\title{
ON A GENERALIZED GREEN'S FUNCTION AND CERTAIN OF ITS APPLICATIONS ${ }^{1}$
}

\author{
STEFAN BERGMAN
}

1. Introduction. A theorem of Blaschke in the theory of a.f. $1 \mathrm{c.v}$. (analytic functions of one complex variable) states: $\sum_{\nu=1}^{\infty} \log \left|a_{\nu}\right|$ $>-\infty,\left|a_{\nu}\right|<1$, is a necessary and sufficient condition for the existence of a non-negative, harmonic function ${ }^{2} H(z), z \in\left[\xi^{2}-\mathcal{S}_{\nu=1}^{\infty}\left\{a_{\nu}\right\}\right]$, $\mathbb{E}^{2}=\varepsilon[|z|<1]$, which possesses the property that $\left[H(z)+\log \left|z-a_{\nu}\right|\right]$, $\nu=1,2, \cdots$, is regular in a neighborhood of $z=a_{\nu}$. By

$$
\exp [-H(z)-i B(z)]=f(z)
$$

where $B(z)$ is a function conjugate to $H(z)$ we obtain a function $f(z)$, $|f(z)| \leqq 1, z \in \mathbb{S}^{2}$, which possesses factors $\left(z-a_{\nu}\right), \nu=1,2, \cdots$.

If one wishes to obtain an analogous result in the theory of a.f. 2 c.v. one must bear in mind at first the following fact:

If $f(z) / g(z)$ is regular in $\mathbb{5}^{2}$ we call $g(z)$ a zero function of $f$ in $\mathbb{5}^{2}$.

Since every function $f$ regular in $\mathbb{F}^{2}$ can be represented in the form $f(z)=\prod\left(z-a_{\nu}\right) k_{\nu}(z)$ where $k_{\nu}(z)$ are regular and nonvanishing in $\xi^{2}$, we need to consider in the theory of a.f. $1 \mathrm{c.v}$. only linear zero functions. In the case of a.f. $2 \mathrm{c.v}$. we cannot in general represent even polynomials as products of linear functions; therefore, one must use for zero functions not only linear expressions but also arbitrary a.f. 2 c.v. [1, p. 1189]. ${ }^{3}$

Furthermore there is lacking in the theory of a.f. 2 c.v. a theorem analogous to the theorem of Riemann, stating that every simply connected domain possessing at least two boundary points can be transformed conformally into f $^{2}$. We cannot therefore limit ourselves to

\footnotetext{
${ }^{1}$ Presented to the Society, April 27, 1940.

${ }^{2}$ We designate by capital and small letters, respectively, real and complex functions of $z_{k}, z_{k}=x_{k}+i y_{k}$, and manifolds by English letters, where the upper index denotes the dimension of the manifold. We omit this index for four-dimensional manifolds. We denote by $\mathcal{E}[\cdots]$ the set of points whose coordinates satisfy the relations indicated in brackets. $\mathcal{S}$ means the logical sum. A horizontal bar above a letter indicates the closure of the set denoted by the letter.

${ }^{3}$ The numbers in brackets refer to the following papers: Stefan Bergman, 1. Proceedings, Akadem.e van Wetenschappen, Amsterdam, vol. 34 (1932), pp. 1188-1194, 2. Mathematische Annalen, vol. 102 (1934), pp. 324-348, 3. Compositio Mathematica, vol. 3 (1936), pp. 136-173, 4. Compositio Mathematica, vol. 6 (1939), pp. 305-335, 5. Stefan Bergman and Marcinkiewicz, Fundamenta Mathematicae, vol. 33 (1939), pp. 75-94, 6. G. Buler, Bulletin de l'Institute Mathématique de Tomsk, vol. 2 (1939), pp. 164-186, 7. S. Saks, Theory of the Integral, Monografie Matematyczne, vol. 7, Warsaw and Lwów, 1937.
} 
consideration of one domain as in the theory of a.f. 1 c.v., but we must study our problem for an arbitrary domain.

In attempting to generalize to the theory of a.f. 2 c.v. the methods which are bases for the proofs of the theorem of Blaschke and other theorems in the theory of meromorphic functions, until now we have limited ourselves to the consideration of a special class of domains (domains with distinguished boundary surface) $[2, \S 1, \S 3 ; 3$, p. 138]; furthermore it was necessary to introduce instead of harmonic functions an "extended class of functions" which contains biharmonic functions (that is, real or imaginary part of a.f. 2 c.v.). This class possesses various properties analogous to those of harmonic functions, but depends upon the domain [4, p. 306 and p. 319]. In the case of a bicylinder $\xi=\varepsilon\left[\left|z_{k}\right|<r_{k}, k=1,2\right]$, the double harmonic functions, that is, functions $H$ satisfying $\partial^{2} H / \partial x_{k}^{2}+\partial^{2} H / \partial y_{k}^{2}=0, k=1,2$, form the extended class of functions.

2. Functions of the extended class. In the present paper we shall consider a special class of domains, $\mathfrak{M}$, with distinguished boundary surface. Let $h\left(z_{2}, \lambda\right), 0 \leqq \lambda \leqq 2 \pi, h\left(z_{2}, 0\right)=h\left(z_{2}, 2 \pi\right)$, be a continuously differentiable function of $z_{2}, \lambda$, which is for every constant $\lambda$ an analytic function of $z_{2},\left|z_{2}\right| \leqq 1$, such that:

$a^{\circ}$. we have

$$
\min \left|h\left(z_{2}, \lambda\right)\right| \geqq \Delta>0, \quad M^{-1} \leqq|\partial h / \partial \lambda| \leqq M<\infty ;
$$

$\mathrm{b}^{\circ}$. it is possible to represent the curve $\mathfrak{b}^{1}\left(z_{2}\right)=\mathcal{S}_{0 \leqq \lambda \leqq 2 \pi} \mathcal{E}\left[z_{1}=h\left(Z_{2}, \lambda\right)\right.$, $\left.Z_{2}=z_{2}\right],\left|z_{2}\right| \leqq 1$, in polar coordinates $\rho, \omega$ in the form $\rho=\rho\left(\omega ; z_{2}\right)$ where $\rho$ is one-valued, and with the further assumption that

$$
\left|\partial^{\mathrm{H}} \rho / \partial \omega^{\mathrm{H}}\right| \leqq M, \mathrm{H}=1,2, \quad M^{-1} \leqq|\partial \omega / \partial \lambda| \leqq M<\infty .
$$

Let $\mathfrak{B}^{2}\left(z_{2}\right)$ be the region in the plane $Z_{2}=z_{2}$ bounded by $\mathfrak{b}^{1}\left(z_{2}\right)$. (See Figure 1, p. 657.) Thus $\mathfrak{B}^{2}\left(z_{2}\right)=\mathcal{S}_{0 \leqq s \leqq 1} \mathcal{S}_{0<\lambda \leqq 2 \pi} \mathcal{E}\left[z_{1}=\operatorname{sh}\left(Z_{2}, \lambda\right), Z_{2}=z_{2}\right]$. The domain $\mathfrak{M}$ is now defined as $\mathfrak{M}=\mathcal{S}_{\left|z_{2}\right|<1} \mathfrak{B}^{2}\left(z_{2}\right)$. The boundary of $\mathfrak{M}$ consists of two segments of analytic hypersurfaces

$$
\begin{aligned}
& \dot{\mathrm{j}}_{1}^{3}=\mathcal{S}_{0 \leqq \lambda \leqq 2 \pi} \Im^{2}(\lambda), \quad \Im^{2}(\lambda)=\mathcal{E}\left[z_{1}=h\left(z_{2}, \lambda\right),\left|z_{2}\right| \leqq 1\right], \\
& \dot{\mathrm{j}}_{2}^{3}=\mathcal{S}_{0 \leqq \phi_{2} \leqq 2 \pi} \mathfrak{B}^{2}\left[\exp \left(i \phi_{2}\right)\right] .
\end{aligned}
$$

The intersection

$$
\begin{array}{r}
\mathfrak{F}^{2}=\dot{i}_{1}^{3} \cdot \dot{j}_{2}^{3}=\mathcal{S}_{0 \leqq \phi_{2} \leqq 2 \pi} \mathfrak{S}^{1}\left[\exp \left(i \phi_{2}\right)\right]=\mathcal{S}_{0 \leqq \lambda \leqq 2 \pi} \mathcal{S}_{0 \leqq \phi_{2} \leqq 2 \pi} \mathcal{E}\left[z_{1}=h\left(\exp \left(i \phi_{2}\right),\right.\right. \\
\left.\lambda), z_{2}=\exp \left(i \phi_{2}\right)\right]=\mathcal{S}_{0 \leqq \lambda \leqq 2 \pi} \mathfrak{i}^{1}(\lambda),
\end{array}
$$

where $\mathfrak{i}^{1}(\lambda)=\mathcal{S}_{0 \leqq \phi_{2} \leqq 2 \pi} \mathcal{E}\left[z_{1}=h\left(\exp \left(i \phi_{2}\right), \lambda\right), z_{2}=\exp \left(i \phi_{2}\right)\right]$, forms the distinguished boundary surface of $\mathfrak{M}$. 
Let a real function $G\left(\phi_{2}, \lambda\right), 0 \leqq \phi_{2} \leqq 2 \pi, 0 \leqq \lambda \leqq 2 \pi$, be given such that, except for a finite number of values of $\lambda, G\left(\phi_{2}, \lambda\right)$ is continuous in $\phi_{2}$ and $\lambda$ for every $\lambda$, in the range $0 \leqq \phi_{2} \leqq 2 \pi$, except for a finite number of values of $\phi_{2}$ (which can vary with $\lambda$ ) and such that ${ }^{4}$ $\iint\left|G\left(\phi_{2}, \lambda\right)\right| d \lambda d \phi_{2}=c<\infty$.

The function $D\left(z_{1}, z_{2}\right) \equiv D\left(z_{1}, z_{2} ; G ; \mathfrak{M}\right)$ of "extended class $E^{\prime}(\mathfrak{M})$ " is defined in $\mathfrak{M}$ in the following way:

$1^{\circ}$. In every point $\left(z_{1}, z_{2}\right)$ of $\Im^{2}(\lambda)$ we define the value of $D$ in such a manner that $D\left[h\left(z_{2}, \lambda\right), z_{2}\right]$ becomes a harmonic function of $x_{2}, y_{2}$ for $\left|z_{2}\right|<1$, which assumes for $\left|z_{2}\right|=1$ the values $G\left(\phi_{2}, \lambda\right)$;

$2^{\circ}$. In every $\mathfrak{B}^{2}\left(z_{2}\right)$ we define the values of $D$ in such a manner that $D\left(z_{1}, z_{2}\right)$ becomes a harmonic function of $x_{1}, y_{1}$ in $\mathfrak{B}^{2}\left(z_{2}\right)$ which assumes on $\mathfrak{b}^{1}\left(z_{2}\right)$ the values $D\left[h\left(z_{2}, \lambda\right), z_{2}\right]$ defined by $1^{\circ}$. We get for $D$ the integral representation

$$
\begin{gathered}
P_{1}=\frac{1}{2 \pi} \frac{1-\left|w\left(Z_{1}, Z_{2}\right)\right|^{2}}{1+\left|w\left(Z_{1}, Z_{2}\right)\right|^{2}-2\left|w\left(Z_{1}, Z_{2}\right)\right| \cos \left[\chi\left(\lambda ; Z_{2}\right)-\operatorname{arc} w\left(Z_{1}, Z_{2}\right)\right]}, \\
P_{2}=\frac{1}{2 \pi} \frac{1-R_{2}^{2}}{1+R_{2}^{2}-2 R_{2} \cos \left(\Phi_{2}-\phi_{2}\right)}, \\
R_{2} e^{i \Phi 2}=Z_{2},
\end{gathered}
$$

where $w\left(\zeta, Z_{2}\right), w\left(0, Z_{2}\right)=0, w^{\prime}\left(0, Z_{2}\right)>0$, is the function which maps $\mathfrak{B}^{2}\left(Z_{2}\right)$ on the unit circle; $\chi\left(\lambda ; Z_{2}\right)=\operatorname{arc} w\left[h\left(Z_{2}, \lambda\right), Z_{2}\right][4$, p. 319]. $H\left(z_{1}, z_{2}\right)$ is said to be $H \in E(\mathfrak{M})$ if $H\left(z_{1}, z_{2}\right)=\lim _{n \rightarrow \infty} D_{n}^{(1)}\left(z_{1}, z_{2}\right)$ $-\lim _{n \rightarrow \infty} D_{n}^{(2)}\left(z_{1}, z_{2}\right)$ where $D_{n}^{(k)}\left(z_{1}, z_{2}\right)=D\left(z_{1}, z_{2} ; G_{n}^{(k)} ; \mathfrak{M}\right)$ is a set of non-decreasing positive functions described above converging uniformly to a limit function in every closed subregion of $\mathfrak{M}$ and almost everywhere on every $\mathcal{S}_{0 \leqq \phi_{2} \leqq 2 \pi} \mathfrak{b}^{1}\left(r e^{i \phi_{2}}\right), r<1$. We have then by a classical theorem of Lebesgue [7, p. 28]

$$
H(0,0)=\frac{1}{4 \pi^{2}} \iint H\left[h\left(r e^{i \phi_{2}}, \lambda\right), r e^{i \phi_{2}}\right] d \phi_{2} d \chi(\lambda ; 0) .
$$

The function $L\left(z_{1}, z_{2}\right)$ is said to be $L \in l(\mathfrak{M})$ if $L$ can be represented in the form

$$
L\left(z_{1}, z_{2}\right)=\sum_{\nu=1}^{\infty}\left[-\log \left|g_{\nu}\left(z_{1}, z_{2}\right)\right|+H_{\nu}\left(z_{1}, z_{2}\right)\right],
$$

\footnotetext{
${ }^{4}$ Here and in the following $\iint$ means $\int_{\phi_{2}=0}^{2 \pi} \int_{\lambda=0}^{2 \pi}$.
} 
the sum converging uniformly in any closed subregion of $\left[\mathfrak{M}-S_{\nu=1}^{\infty} \mathfrak{S}_{\nu}^{2}\right]$, $\left(\mathfrak{S}_{\nu}^{2}=\varepsilon\left[g_{\nu}\left(z_{1}, z_{2}\right)=0\right]\right.$ and converging almost everywhere on every $\mathcal{S}_{0 \leqq \phi_{2} \leqq 2 \pi} \mathfrak{b}^{1}\left(r e^{i \phi_{2}}\right), r<1$, and such that

$$
\begin{aligned}
& \iint L\left[h\left(r e^{i \phi_{2}}, \lambda\right), r e^{i \phi_{2}}\right] d \phi_{2} d \chi(\lambda ; 0)=c_{r} \geqq-c_{L}>-\infty, r<1 ; \\
& \iint\left|L\left[h\left(r e^{i \phi_{2}}, \lambda\right), r e^{i \phi_{2}}\right]\right| d \phi_{2} d \chi(\lambda, 0)<\infty, \quad r<1 .
\end{aligned}
$$

$g_{\nu}\left(z_{1}, z_{2}\right)$ are a.f. 2 c.v. regular in $\overline{\mathfrak{M}}$ and $H_{\nu}\left(z_{1}, z_{2}\right) \in E(\mathfrak{M})$.

In an analogous manner we introduce the classes $E^{\prime}\left(\mathfrak{M}_{r}\right), E\left(\mathfrak{M}_{r}\right)$, $l\left(\mathfrak{M}_{r}\right), \mathfrak{M}_{r}=\mathcal{S}_{\left|z_{2}\right|<r} \mathfrak{B}^{2}\left(z_{2}\right)=\varepsilon\left[\left|z_{2}\right|<r,\left(z_{1}, z_{2}\right) \in \mathfrak{B}^{2}\left(z_{2}\right)\right]$.

REMARK 1. Since $\mathcal{E}\left[z_{1}=h\left(z_{2}, \lambda\right),\left|z_{2}\right|<r\right] \subset \Im^{2}(\lambda)[$ cf. (2.3)] every function $H\left(z_{1}, z_{2}\right)$ which is of class $E^{\prime}(\mathfrak{M})$ or $E(\mathfrak{M})$ belongs to $E^{\prime}\left(\mathfrak{M}_{r}\right)$ or $E\left(\mathfrak{M}_{r}\right)$ respectively.

3. Lemmas. For the proof of the main theorem we need several lemmas.

I. There exists a function $\alpha_{r}\left(Z_{1}, Z_{2}\right), \alpha_{r}\left(Z_{1}, Z_{2}\right)<\infty$ for $\left(Z_{1}, Z_{2}\right) \in \mathfrak{M}_{r}$, which depends only on $\mathfrak{M}_{r}$ such that for every non-negative $H\left(Z_{1}, Z_{2}\right)$ $\in E\left(\mathfrak{M}_{r}\right)$ we have

$$
H\left(Z_{1}, Z_{2}\right) \leqq \alpha_{r}\left(Z_{1}, Z_{2}\right) H(0,0) .
$$

Proof. According to (2.4) we have for every non-negative $D_{n}\left(Z_{1}, Z_{2}\right) \in E^{\prime}\left(\mathfrak{M}_{r}\right)$

$$
D\left(Z_{1}, Z_{2}\right) \leqq \frac{r+R_{2}}{r-R_{2}} \frac{1+\left|w\left(Z_{1} ; Z_{2}\right)\right|}{1-\left|w\left(Z_{1} ; Z_{2}\right)\right|} \iint G\left(\phi_{2}, \lambda\right) d \phi_{2} d \chi\left(\lambda ; Z_{2}\right) .
$$

Since $\mathfrak{b}^{1}\left(Z_{2}\right)$ satisfies the conditions $\mathrm{a}^{\circ}$ and $\mathrm{b}^{\circ}$ for every $Z_{2}$, we obtain by Lemma 7 of $[5]$

$$
\begin{aligned}
M_{1}^{-1} d \operatorname{arc} w[h(0, \lambda), 0] & \leqq d \operatorname{arc} w\left[h\left(Z_{2}, \lambda\right), Z_{2}\right] \equiv d \chi\left(\lambda, Z_{2}\right) \\
& \leqq M_{1} d \operatorname{arc} w[h(0, \lambda), 0] \equiv M_{1} d \chi(\lambda ; 0),
\end{aligned}
$$

$M_{1}$ being an appropriate constant. By Lemma 5 of $[5],,^{5}$ (3.2) and (2.4) we obtain (3.1) with $H$ replaced by $D$ and with $\alpha_{r}\left(Z_{1}, Z_{2}\right)$ $=\left(r+R_{2}\right)\left(r-R_{2}\right)^{-1}\left[1+\left|w\left(Z_{1}, Z_{2}\right)\right|\right]\left[\delta\left(Z_{1}, Z_{2}\right)\right]^{-1}, \delta\left(Z_{1}, Z_{2}\right)$ being an appropriate constant. Since we can approximate $H \in E\left(\mathfrak{M}_{r}\right)$ uniformly in any closed subregion of $\mathfrak{M}_{r}$ by an appropriate $D\left(Z_{1}, Z_{2}\right)=D_{n}^{(1)}\left(Z_{1}, Z_{2}\right)$ $-D_{n}^{(2)}\left(Z_{1}, Z_{2}\right), D_{n}^{(k)}\left(Z_{1}, Z_{2}\right) \in E^{\prime}\left(\mathfrak{M}_{r}\right),(3.1)$ is valid for $H\left(Z_{1}, Z_{2}\right)$.

${ }^{5}$ In the Lemma 5 of [5] $\Phi(z)$ is assumed to vanish at $z=0$. 


$$
\begin{array}{r}
\Gamma\left(z_{1}, z_{2} ; g ; \mathfrak{M}_{r}\right)=-\log \left|g\left(z_{1}, z_{2}\right)\right|+D\left(z_{1}, z_{2} ; \log |g| ; \mathfrak{M}_{r}\right), \\
g \equiv g\left[h\left(r e^{i \phi_{2}}, \lambda\right), r e^{i \phi_{2}}\right],
\end{array}
$$

is designated Green's function of the extended class corresponding to $g$ and $\mathfrak{M}_{r}, \mathfrak{M}_{1}=\mathfrak{M}$. Here $g$ is supposed to be regular in $\overline{\mathfrak{M}}$.

In every point $\left(z_{1}, z_{2}\right) \in \mathfrak{F}_{r}^{2}=\mathcal{S}_{0 \leqq \phi_{2} \leqq 2 \pi} \mathfrak{b}^{1}\left(r e^{i \phi_{2}}\right)$ where $g\left(z_{1}, z_{2}\right) \neq 0$, $\Gamma\left(z_{1}, z_{2} ; g ; \mathfrak{M}_{r}\right)=0$.

Since the set of points of $\mathfrak{F}_{r}^{2}$ where $g\left(z_{1}, z_{2}\right)=0$ has zero measure, we have

$$
\iint \Gamma\left[h\left(r e^{i \phi_{2}}, \lambda\right), r e^{i \phi_{2}} ; g ; \mathfrak{M}_{r}\right] d \phi_{2} d \chi(\lambda ; 0)=0 .
$$

In order to avoid tedious operations in our later considerations we suppose in the following that $g$ and $g_{\nu}$ have no factors of the form $\left[z_{1}-h\left(z_{2}, \lambda\right)\right], \lambda$ const.

II. For $\left(z_{1}, z_{2}\right) \in \mathfrak{M}+\mathfrak{i}_{1}^{3}$,

$$
\Gamma\left(z_{1}, z_{2} ; g ; \mathfrak{M}\right) \geqq 0 .
$$

Proof. Since in every $\mathfrak{B}^{2}\left(z_{2}^{0}\right),\left|z_{2}^{0}\right|<1, \Gamma\left(z_{1}, z_{2} ; g ; \mathfrak{M}\right)$ is a non-negative harmonic function of $x_{1}, y_{1}$, which may become infinite at a finite number of points $a$ like $-p \log \left|z_{1}-a\right|, p$ positive integer, it is necessary to show only that $\Gamma\left(z_{1}, z_{2} ; g ; \mathfrak{M}\right) \geqq 0$ for $\left(z_{1}, z_{2}\right) \in \mathrm{j}_{1}^{3}$ $=\mathcal{S}_{\left|z_{2}\right|<1} \mathfrak{b}^{1}\left(z_{2}\right)=\mathcal{S}_{0 \leqq \lambda \leqq 2 \pi} \mathfrak{S}^{2}(\lambda)$. (The other possibility is that $g$ has a factor $\left(z_{2}-a\right)$, but then $\Gamma\left(z_{1}, a ; g ; \mathfrak{M}\right)=+\infty$.) In every $\mathfrak{S}^{2}(\lambda)$ we have

$$
\begin{aligned}
& \Gamma\left(z_{1}, z_{2} ; g ; \mathfrak{M}\right)=\Gamma\left[h\left(z_{2}, \lambda\right), z_{2} ; g ; \mathfrak{M}\right] \\
& \quad=-\log \left|g\left[h\left(z_{2}, \lambda\right), z_{2}\right]\right|+D\left[h\left(z_{2}, \lambda\right), z_{2} ; \log |g| ; \mathfrak{M}\right] .
\end{aligned}
$$

$\Gamma\left[h\left(z_{2}, \lambda\right), z_{2} ; g ; \mathfrak{M}\right]$ is for every $\lambda$ a harmonic function of $x_{2}, y_{2}$ in $\left|z_{2}\right|<1$ except at a finite number of points in which it becomes $\infty$ and which assumes the boundary values 0 on $\left|z_{2}\right|=1$. The function (3.7) is obviously non-negative in $\left|z_{2}\right|<1$.

III. The following relation holds:

$$
\lim _{r \rightarrow 1} \Gamma\left(0,0 ; g ; \mathfrak{M}_{r}\right)=\Gamma(0,0 ; g ; \mathfrak{M}) \text {. }
$$

Proof. In order to prove (3.8) we shall show that

$$
\lim _{r \rightarrow 1} D\left(0,0 ; \log |g|, \mathfrak{M}_{r}\right)=D(0,0 ; \log |g| ; \mathfrak{M}) .
$$


Since $g\left(z_{1}, z_{2}\right)$ is regular on $\mathfrak{F}_{1}^{2}=\mathcal{S}_{0 \leqq \phi_{2} \leqq 2 \pi} \mathfrak{b}^{1}\left(e^{i \phi_{2}}\right), \mathfrak{F}_{1}^{2}=\mathfrak{F}^{2}$, we can find by Weierstrass' preparation theorem and the Heine-Borel theorem a finite number of domains $\mathfrak{S}_{k}=\mathcal{E}\left[\left|z_{\mathrm{H}}-\zeta_{\mathrm{H}}^{(k)}\right| \leqq \rho, \mathrm{H}=1,2\right], k=1, \cdots, n$, $\left(\zeta_{1}^{(k)}, \zeta_{2}^{(k)}\right) \in \mathfrak{F}_{1}^{2}$, with the following properties:

$1^{\circ}$. $\mathfrak{S}_{k=1}^{n} \mathfrak{S}_{k}$ covers the total (four-dimensional) neighborhood of $\mathfrak{F}_{1}^{2}$;

$2^{\circ}$. in every $\mathfrak{S}_{k}$,

$$
g=\left(z_{2}-\zeta_{2}^{(k)}\right)^{\mu_{k}} \prod_{\mathbf{H}=1}^{p_{k}}\left[z_{1}-\alpha_{\mathrm{H}}^{(k)}\left(z_{2}\right)\right] \Psi_{k}\left(z_{1}, z_{2}\right)
$$

where $p_{k}, \mu_{k}$ are non-negative integers, $\Psi_{k}$ is an a.f. 2 c.v. regular and nonvanishing in $\mathfrak{S}_{k}$ and $\alpha_{\mathrm{H}}^{(k)}\left(z_{2}\right), \alpha_{\mathrm{H}}^{(k)}\left(\zeta_{2}^{(k)}\right)=\zeta_{1}^{(k)}$ are algebroid ${ }^{6}$ in a certain neighborhood of $z_{2}=\zeta_{2}^{(k)}$.

We can suppose that $r_{0}$ is chosen so near to 1 that $g$ has no factors $\left(z_{2}-r e^{i \phi_{2}}\right), r_{0} \leqq r<1$. Hence in order to prove (3.9) it is sufficient to prove that

$$
\iint \log \left|z_{1}-\alpha_{\mathrm{H}}^{(k)}\left(z_{2}\right)\right| d \phi_{2} d \chi(\lambda ; 0), \quad z_{2}=r e^{i \phi_{2}},
$$

is a continuous function of $r$ for every $k$ and $H$, the in tegration being

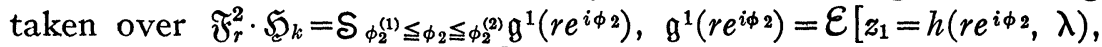
$\left.z_{2}=r e^{i \phi_{2}}, \lambda_{1}\left(r, \phi_{2}\right) \leqq \lambda \leqq \lambda_{2}\left(r, \phi_{2}\right)\right]$.

Let $\delta>0, \epsilon>0$ and $\mathfrak{R}_{\delta}^{2}=\mathcal{E}\left[\left|z_{1}-\zeta_{1}^{(k)}\right| \leqq \delta, z_{2}=r e^{i \phi_{2}}\right]$. We can determine an $r_{0}$ so near to 1 that for $r_{0} \leqq r \leqq 1$ and $\psi-\epsilon \leqq \phi_{2} \leqq \psi+\epsilon$ where $\psi=\operatorname{arc} \zeta_{2}^{(k)}, \alpha_{\mathrm{H}}^{(k)}\left(r e^{i \phi_{2}}\right) \operatorname{lies}^{7}$ in $\Omega_{\delta}^{2}$ and the point $\alpha_{\mathrm{H}}^{(k)}\left(r e^{i \phi 2}\right)$ is not contained in $\mathfrak{g}^{1}\left(r e^{i \phi 2}\right)$ for $r_{0} \leqq r \leqq 1, \phi_{2}^{(1)} \leqq \phi_{2} \leqq \psi-\epsilon$ or $\psi+\epsilon \leqq \phi_{2} \leqq \phi_{2}^{(2)}$.

We dissect every $\mathfrak{g}^{1}\left(r e^{i \phi_{2}}\right), r_{0} \leqq r \leqq 1, \psi-\epsilon \leqq \phi_{2} \leqq \psi+\epsilon$, into two parts; $\mathfrak{g}_{\delta}^{1}\left(r e^{i \phi_{2}}\right)=\Omega_{\delta}^{2} \cdot \mathfrak{g}^{1}\left(r e^{i \phi_{2}}\right)=\varepsilon\left[z_{1}=h\left(z_{2}, \lambda\right), z_{2}=r e^{i \phi_{2}}, \lambda^{(1)}\left(\delta, \phi_{2}\right) \leqq \lambda\right.$ $\left.\leqq \lambda^{(2)}\left(\delta, \phi_{2}\right)\right]$ and $\mathfrak{f}^{1}\left(r e^{i \phi_{2}}\right)=\mathfrak{g}^{1}\left(r e^{i \phi_{2}}\right)-g_{\delta}^{1}\left(r e^{i \phi_{2}}\right)$.

(3.10) Taken over $\mathcal{S}_{\phi_{2}^{(1)} \leqq \phi_{2} \leqq \psi-\epsilon} \mathfrak{g}^{1}\left(r e^{i \phi_{2}}\right), \quad \mathcal{S}_{\psi+\epsilon \geqq \phi_{2} \leqq \phi_{2}^{(2)}} \mathfrak{g}^{1}\left(r e^{i \phi_{2}}\right)$ or

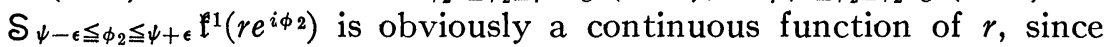
$\alpha_{\mathbf{H}}^{(k)}\left(r e^{i \phi_{2}}\right)$ does not lie on these surfaces. By (2.1) and (2.2) there exists by Lemmas 3 and 4 of $[5]$ a $\mathrm{P}=\mathrm{P}(M, \Delta)$ such that every circle of radius $\mathrm{P}$, tangent to $\mathfrak{b}^{1}\left(r e^{i \phi_{2}}\right)$ at an arbitrary point $h\left(r e^{i \phi 2}, \lambda\right)$ of $\mathfrak{b}^{1}\left(r e^{i \phi_{2}}\right)$ lies either entirely inside of $\mathfrak{B}^{2}\left(r e^{i \phi_{2}}\right)$ or outside of this domain.

${ }^{6}$ A function $\alpha=\alpha(z)$ satisfying the equation $\alpha^{m}+g_{1}(z) \alpha^{m-1}+\cdots+g_{m}(z)=0$, where $g_{p}(z), p=1,2, \cdots, m$, are a.f. of 1 c.v. regular in the domain $\mathfrak{U}^{2}$ is said to be "algebroid in $\mathfrak{U}^{2} . "$

${ }^{7}$ Here and in the following part of this section we write for the sake of shortness $h\left(r e^{i \phi_{2}}, \lambda\right)$ instead of $\left[h\left(r e^{i \phi_{2}}, \lambda\right), r e^{i \phi_{2}}\right]$ and $\alpha_{\mathrm{H}}^{(k)}\left(r e^{i \phi_{2}}\right)$ instead of $\left[\alpha_{\mathrm{H}}^{(k)}\left(r e^{\left.i \phi_{2}\right)}, r e^{i \phi_{2}}\right]\right.$. (The points are supposed to be points of the space $\left(z_{1}, z_{2}\right)$, lying in the plane $z_{2}=r e^{i \phi_{2}}$, but we omit the coordinate $z_{2}$.) 
If therefore $\min _{\lambda}\left|\alpha_{\mathrm{H}}^{(k)}\left(r e^{i \phi_{2}}\right)-h\left(r e^{i \phi_{2}}, \lambda\right)\right|<\mathrm{P}$ and if $h\left(r e^{i \phi_{2}}, \lambda^{0}\right)$ is the point of $\mathfrak{b}^{1}\left(r e^{i \phi_{2}}\right)$ which is nearest to $\alpha_{\mathrm{H}}^{(k)}\left(r e^{i \phi_{2}}\right)$, then $\alpha_{\mathrm{H}}^{(k)}\left(r e^{i \phi_{2}}\right)$ lies on the normal $\mathfrak{n}^{1}\left(r e^{i \phi_{2}}\right)$ to $\mathfrak{b}^{1}\left(r e^{i \phi_{2}}\right)$ at $h\left(r e^{i \phi_{2}}, \lambda^{0}\right)$ which lies in the plane $\zeta_{2}=z_{2}$.

It follows by (2.1) and (2.2) that $h\left(r e^{i \phi_{2}}, \lambda\right)$ lies, for $\left|\lambda-\lambda^{0}\right| \leqq \mathrm{P} M^{-1}$ inside of the triangle $\mathfrak{I}^{2}$, the sides of which are formed by arcs of the

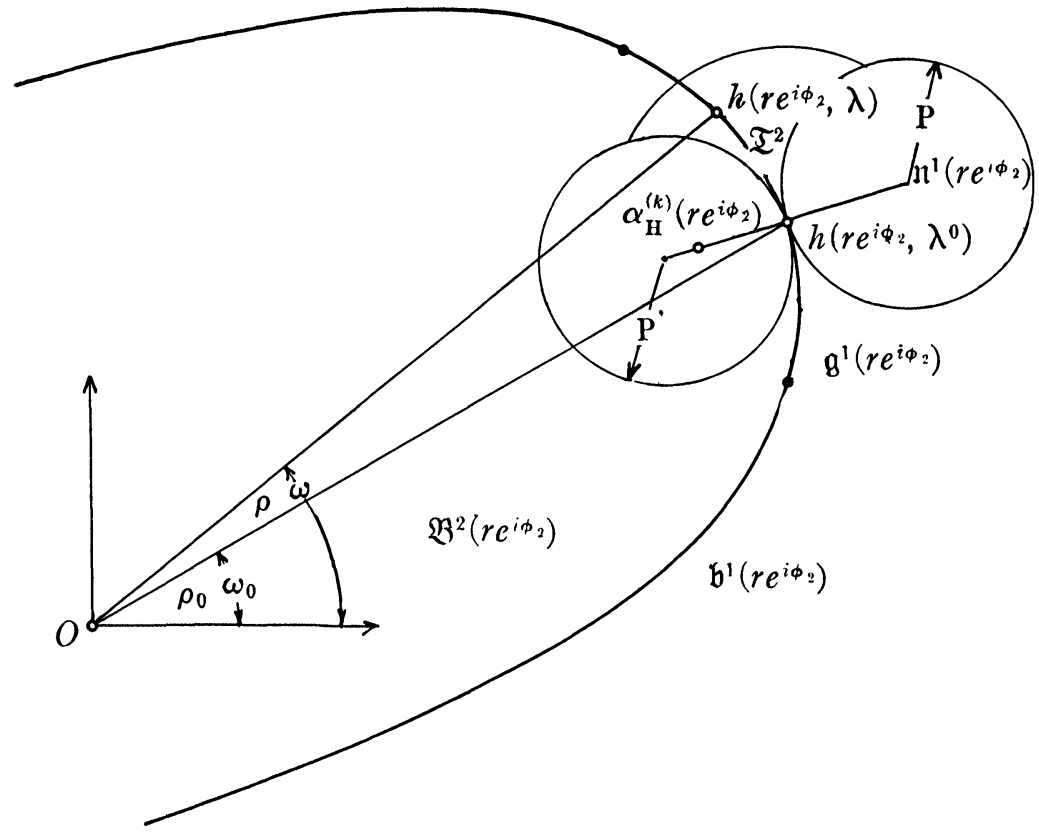

exterior and of the interior circles of radius $\mathrm{P}$, tangent to $\mathfrak{b}^{1}\left(r e^{i \phi 2}\right)$ at $h\left(r e^{i \phi 2}, \lambda^{0}\right)$ and of the circle $\left|z_{1}-h\left(r e^{i \phi 2}, \lambda^{0}\right)\right|=2^{1 / 2} \mathrm{P}$.

The straight line passing through $h\left(r e^{i \phi_{2}}, \lambda\right)$ and $h\left(r e^{i \phi_{2}}, \lambda^{0}\right)$ forms with $\mathfrak{n}^{1}\left(r e^{i \phi_{2}}\right)$ an angle $\beta$, for which $45^{\circ} \leqq|\beta| \leqq 135^{\circ}$.

Since by (2.1) and (2.2) the length of $\left[h\left(r e^{i \phi 2}, \lambda\right), h\left(r e^{i \phi 2}, \lambda^{0}\right)\right]$ is not less than

$$
\begin{gathered}
\left|\rho e^{i \omega}-\rho_{0} e^{i \omega_{0}}\right| \geqq \frac{1}{2} \Delta\left|\omega-\omega_{0}\right| \geqq \frac{1}{2} \Delta \mathrm{M}^{-1} \cdot\left|\lambda-\lambda^{0}\right|, \\
\left.\rho e^{i \omega} \equiv h\left(r e^{i \phi_{2}}, \lambda\right), \rho_{0} e^{i \omega_{0}} \equiv h\left(r e^{i \phi_{2}}, \lambda^{0}\right),\left[\sin \frac{1}{2}\left(\omega-\omega^{0}\right)\right] / \omega-\omega_{0}>\frac{1}{4}\right],
\end{gathered}
$$

the distance between $h\left(r e^{i \phi_{2}}, \lambda\right)$ and $\mathfrak{n}^{1}\left(r e^{i \phi_{2}}\right)$ and therefore between $h\left(r e^{i \phi_{2}}, \lambda\right)$ and $\alpha_{\mathbf{H}}^{(k)}\left(r e^{i \phi_{2}}\right)\left[\mathfrak{\epsilon n}^{1}\left(r e^{i \phi_{2}}\right)\right]$ is not less than

$$
\frac{1}{2}\left|\lambda-\lambda^{0}\right| \Delta M^{-1} \sin 45^{\circ}=\left(\frac{1}{2}\right)^{3 / 2}\left|\lambda-\lambda^{0}\right| \Delta M^{-1} \text {. }
$$


If $\left|\lambda-\lambda^{0}\right|>\mathrm{P} M^{-1}$, then we have the two following possibilities:

$1^{\circ}$. $h\left(r e^{i \phi_{2}}, \lambda\right)$ lies in the triangle $\mathfrak{T}^{2}$ and we have by $(2.2)$

$$
\left|\omega-\omega^{0}\right| \geqq \mathrm{P} M^{-2} \text {, }
$$

and from this $\left|h\left(r e^{i \phi_{2}}, \lambda\right)-\alpha_{\mathbf{H}}^{(k)}\left(r e^{i \phi_{2}}\right)\right| \geqq\left(\frac{1}{2}\right)^{3 / 2} \Delta \mathrm{P} M^{-2}$, since $h\left(r e^{i \phi_{2}}\right.$, $\lambda) \in \mathfrak{I}^{2}$;

$2^{\circ} . h\left(r e^{i \phi_{2}}, \lambda\right)$ lies outside of $\mathfrak{I}^{2}$ and then, since $\alpha_{\mathrm{H}}^{(k)}\left(r e^{i \phi_{2}}\right)$ lies between $h\left(r e^{i \phi_{2}}, \lambda^{0}\right)$ and the center of the circle, we have

$$
\left|h\left(r e^{i \phi_{2}}, \lambda\right)-\alpha_{\mathrm{H}}^{(k)}\left(r e^{i \phi 2}\right)\right| \geqq \mathrm{P} \text {. }
$$

Since we can suppose that $M \geqq 1$ and $\mathrm{P} \leqq 1$ we have finally that

$$
\left|h\left(r e^{i \phi_{2}}, \lambda\right)-\alpha_{\mathrm{H}}^{(k)}\left(r e^{i \phi_{2}}\right)\right| \geqq\left(\frac{1}{2}\right)^{3 / 2} \Delta \mathrm{P} M^{-1} \cdot\left|\lambda-\lambda^{0}\right|_{M^{-1}}
$$

where $\left|\lambda-\lambda^{0}\right|_{M^{-1}}$ is $\left|\lambda-\lambda^{0}\right|$ if $\left|\lambda-\lambda^{0}\right| \leqq M^{-1}$, and is $M^{-1}$ if $\left|\lambda-\lambda^{0}\right|$ $\geqq M^{-1}$.

Thus the integral (3.10) taken over $\mathcal{S}_{\psi-\epsilon \leqq \phi_{2} \leqq \psi+\epsilon} \mathfrak{g}_{\delta}^{1}\left(r e^{i \phi_{2}}\right)$ is smaller in absolute value than

$$
M \int_{\psi-\epsilon}^{\psi+\epsilon} \int_{\lambda_{(1)}^{\left.(1), \phi_{2}\right)}}^{\lambda^{(2)}\left(\delta, \phi_{2}\right)} \mid \log \left(\left(\frac{1}{2}\right)^{3 / 2} \Delta P M^{-1} \cdot\left|\lambda-\lambda^{0}\right|_{\left.M^{-1}\right)} \mid d \lambda d \phi_{2}\right.
$$

which converges to 0 as $\delta \rightarrow 0$.

4. The main theorem. Let $\left\{g_{\nu}\left(z_{1}, z_{2}\right)\right\}$ designate a set of a.f. 2 c.v. regular in $\mathfrak{M}$ and possessing the property: A. For every $r<1$ there exists an $N(r)$ so that $g_{\nu}\left(z_{1}, z_{2}\right), \nu>N(r)$, does not vanish in $\overline{\mathfrak{M}}_{r}=\mathcal{S}_{\left|z_{2}\right| \leqq r \bar{B}^{2}}\left(z_{2}\right)$. Then

$$
\sum_{\nu=1}^{\infty} \Gamma\left(0,0 ; g_{\nu} ; \mathfrak{M}\right)<\infty
$$

is a necessary and sufficient condition for the existence of a function $F\left(z_{1}, z_{2}\right) \in l(\mathfrak{M}), F(0,0)<\infty$, which possesses the property that for every $\nu$ $F\left(z_{1}, z_{2}\right)+\log \left|g_{\nu}\left(z_{1}, z_{2}\right)\right|$ is regular on $\$_{\nu}^{2}$ except at the intersection points (\$) ${ }_{\nu}^{2} \cdot \$_{n}^{2}, n \neq \nu$.

Proof. (4.1) is necessary. The function $F$ becomes infinite like $-\log \left|g_{\nu}\left(z_{1}, z_{2}\right)\right|$ on $\mathbb{S S}_{\nu}^{2}, \nu=1,2, \ldots$. Since by A, $\mathfrak{S H}_{\nu}^{2}$ for $\nu>N(r)$ lies outside of $\mathfrak{M}_{r}$ and therefore a fortiori outside of $\mathfrak{M}_{\rho}, \quad \rho \leqq r$, $F\left(z_{1}, z_{2}\right)-\sum_{\nu=1}^{N(r)} \Gamma\left(z_{1}, z_{2} ; g_{\nu} ; \mathfrak{M}_{\rho}\right)$ has no singularities in $\mathfrak{M}_{\rho}$. Since $-\log \left|g_{\nu}\left(z_{1}, z_{2}\right)\right|$ for $\nu>N(r)$ are regular biharmonic functions in $\overline{\mathfrak{M}}_{\rho}$ we have by Remark 1 that $\left[-\log \left|g_{\nu}\left(z_{1}, z_{2}\right)\right|+H_{\nu}\left(z_{1}, z_{2}\right)\right] \in E\left(\mathfrak{M l}_{\rho}\right)$ for $\nu>N(r)$ and therefore that 


$$
\begin{aligned}
\iint \sum_{\nu=N(r)+1}^{m}\left[-\log \left|g_{\nu}\right|+H_{\nu}\right] d \psi_{2} d \chi(\lambda ; 0) \\
\quad=\frac{1}{4 \pi^{2}} \sum_{\nu=N(r)+1}^{m}\left[-\log \left|g_{\nu}(0,0)\right|+H_{\nu}(0,0)\right] .
\end{aligned}
$$

Since

$$
\begin{aligned}
F\left(z_{1}, z_{2}\right) & -\sum_{\nu=1}^{N(r)} \Gamma\left(z_{1}, z_{2} ; g_{\nu} ; \mathfrak{M}_{\rho}\right) \\
& =\lim _{m \rightarrow \infty} \sum_{\nu=N(r)+1}^{m}\left[-\log \left|g_{\nu}\left(z_{1}, z_{2}\right)\right|+H_{\nu}\left(z_{1}, z_{2}\right)\right]+P\left(z_{1}, z_{2}\right)
\end{aligned}
$$

is absolutely integrable, where

$$
P\left(z_{1}, z_{2}\right)=\sum_{\nu=1}^{N(r)}\left[H_{\nu}\left(z_{1}, z_{2}\right)-D\left(z_{1}, z_{2} ; g_{\nu}, \mathfrak{M}_{\rho}\right)\right]
$$

[cf. (2.6), (2.8), and (3.4)], it follows by (4.2), (4.3) and the Lebesgue theorem $\left[7\right.$, p. 29] that ${ }^{8}$

$$
\begin{aligned}
\iint & F d \phi_{2} d \chi(\lambda ; 0)=\iint\left[F-\sum_{\nu=1}^{N(r)} \Gamma_{\nu \rho}\right] d \phi_{2} d \chi(\lambda ; 0) \\
= & \iint\left[\sum_{\nu=N(r)+1}^{\infty}\left(-\log \left|g_{\nu}\right|+H_{\nu}\right)+P\right] d \phi_{2} d \chi(\lambda ; 0) \\
= & \frac{1}{4 \pi^{2}}\left\{\sum_{\nu=N(r)+1}^{\infty}\left[-\log \left|g_{\nu}(0,0)\right|+H_{\nu}(0,0)\right]+P(0,0)\right\} \\
= & \frac{1}{4 \pi^{2}}\left[F(0,0)-\sum_{\nu=1}^{N(r)} \Gamma\left(0,0 ; g_{\nu} ; \mathfrak{M}_{\rho}\right)\right] .
\end{aligned}
$$

By (2.7), (3.6) the right-hand member is $\geqq-C_{F}$ and therefore for $t \leqq N(r)$ we have by II

$$
\sum_{\nu=1}^{t} \Gamma\left(0,0 ; g_{\nu} ; \mathfrak{M}_{\rho}\right) \leqq 4 \pi^{2} C_{F}+F(0,0) .
$$

By Lemma III we can replace in (4.5) $\mathfrak{M}_{\rho}$ by $\mathfrak{M}$ and since this inequality is true for every $t,(4.1)$ holds.

In order to prove that (4.1) is sufficient we shall show that $\sum_{\nu=1}^{\infty} \Gamma\left(z_{1}, z_{2} ; g_{\nu} ; \mathfrak{M}\right) \in l(\mathfrak{M})$. For $r<1, N(r)<m<\infty$,

${ }^{8}$ The arguments of $g_{\nu}, H_{\nu}, P, \Gamma_{\nu \rho}$ lacking in (4.2) and (4.4) are assumed to be $\left[h\left(\rho e^{i \phi_{2}}, \lambda\right), \rho e^{i \phi_{2}}\right]$.

$\Gamma_{\nu \rho}$ in $(4.4)$ is $\Gamma\left[h\left(\rho e^{i \phi_{2}}, \lambda\right), \rho e^{i \phi_{2}} ; g_{\nu} ; \mathfrak{M} \mathcal{M}_{\rho}\right]$. 


$$
\sum_{\nu=N(r)+1}^{m} \Gamma\left(z_{1}, z_{2} ; g_{\nu} ; \mathfrak{M}\right) \in E^{\prime}\left(\mathfrak{M}_{r}\right)
$$

By II and I we have

$$
\sum_{\nu=N(r)+1}^{m} \Gamma\left(z_{1}, z_{2} ; g_{\nu} ; \mathfrak{M}\right) \leqq \alpha_{r}\left(z_{1}, z_{2}\right) \sum_{\nu=N(r)+1}^{m} \Gamma\left(0,0 ; g_{\nu} ; \mathfrak{M}\right)
$$

Therefore by II and $(4.1), \sum_{\nu=N(r)+1}^{\infty} \Gamma\left(z_{1}, z_{2} ; g_{\nu} ; \mathfrak{M}\right)$ converges uniformly in any closed subregion of $\mathfrak{M}$.

Since

$$
\begin{aligned}
\iint\left\{\sum_{\nu=N(r)+1}^{m} \Gamma\left[h\left(r e^{i \phi_{2}}, \lambda\right), r e^{i \phi_{2}} ; g_{\nu} ; \mathfrak{M}\right]\right\} d \phi_{2} d \chi(\lambda ; 0) \\
=\frac{1}{4 \pi^{2}} \sum_{\nu=N(r)+1}^{m} \Gamma\left(0,0 ; g_{\nu} ; \mathfrak{M}\right),
\end{aligned}
$$

by II and the known theorem of Lebesgue $[7$, p. 28]

$$
\sum_{\nu=N(r)+1}^{\infty} \Gamma\left(z_{1}, z_{2} ; g_{\nu} ; \mathfrak{M}\right)
$$

exists almost everywhere on $\mathfrak{F}_{r}^{2}, r<1$, and we have

$$
\begin{aligned}
\iint\left\{\sum_{\nu=N(r)+1}^{\infty} \Gamma\left[h\left(r e^{i \phi_{2}}, \lambda\right), r e^{i \phi_{2}} ; g_{\nu} ; \mathfrak{M}\right]\right\} d \phi d \chi(\lambda 0) & \\
& =\frac{1}{4 \pi^{2}} \sum_{\nu=N(r)+1}^{\infty} \Gamma\left(0,0 ; g_{\nu} ; \mathfrak{M}\right) .
\end{aligned}
$$

Therefore by (4.1) $F\left(z_{1}, z_{2}\right)=\sum_{\nu=1}^{\infty} \Gamma\left(z_{1}, z_{2} ; g_{\nu} ; \mathfrak{M}\right)$ exists in $\mathfrak{M}=\lim _{r \rightarrow 1} \mathfrak{M}_{r}$. It follows by II that $F\left(z_{1}, z_{2}\right) \geqq 0,\left(z_{1}, z_{2}\right) \in \mathfrak{M}+\mathfrak{i}_{1}^{3}$, and by (4.8) and (4.1) we have that

$$
\iint\left\{F\left[h\left(r e^{i \phi_{2}}, \lambda\right), r e^{i \phi_{2}}\right]\right\} d \phi_{2} d \chi(\lambda ; 0) \geqq 0, \quad r<1 .
$$

REMARK. Since double harmonic functions are the extended class for any bicylinder, an analogous procedure shows that in the case of a bicylinder $\mathcal{E}\left[\left|z_{k}\right|<1, k=1,2\right]$ A (see p. 658) can be replaced by the (weaker) hypothesis that $g_{\nu}\left(z_{1}, z_{2}\right), \nu \geqq N(r)$, do not vanish in $\varepsilon\left[\left|z_{k}\right| \leqq r, k=1,2\right]$. Furthermore we wish to notice that from results of Buler [6] one can obtain in this case certain properties of lines $\mathcal{E}\left[F\left(z_{1}, z_{2}\right)=0,\left|z_{1}\right|=A\left|z_{2}\right| \alpha, A, \alpha>0,\left|z_{k}\right|<1\right]$ if $F$ is biharmonic in $\left[\mathfrak{E}-S_{\nu=1}^{\infty}\left(\mathfrak{S}_{\nu}^{2}\right]\right.$. 
Finally we wish to indicate that a procedure analogous to those of [4] enables us to associate with every function $f$, meromorphic in $\mathfrak{M}$, a characteristic function $\mathrm{T}(r, f), r<1$. Using the results of [5] and those of a work of Bers ${ }^{9}$ as well as the theorem of this paper it is possible to show that, under certain hypotheses, $|f|$ possesses boundary values almost everywhere on $\mathfrak{F}^{2}$, if the $\mathrm{T}(r, f)$ is uniformly bounded as $r \rightarrow 1$.

\title{
Massachusetts Institute of Technology
}

${ }^{9}$ The paper of Bers will appear in American Journal of Mathematics. A preliminary report of his work may be found in Comptes Rendus de l'Académie des Sciences, Paris, vol. 208 (1939), pp. 1273-1275 and 1475-1477.

\section{MONOTONIC COLLECTIONS OF PERIPHERALLY SEPARABLE CONNECTED DOMAINS ${ }^{1}$}

\author{
F. B. JONES
}

In my vain attempts to construct an example of a Moore space which is normal but not metric, ${ }^{2}$ I have discovered a few simple and useful theorems about metric spaces which sound familiar but surprisingly do not seem to be known or in the literature. The following is such a theorem and deals with certain conditions under which a monotonic collection of domains contains a countable monotonic subcollection running upward through it. Application of the theorem to certain well ordered sequences is immediate.

Definitions. ${ }^{3}$ A collection $G$ of point sets is said to be monotonic provided that if $g_{1}$ and $g_{2}$ are elements of $G$ then either $g_{1}$ contains $g_{2}$ or $g_{2}$ contains $g_{1}$. A subcollection $H$ of a collection $G$ of point sets is said to run upward through $G$ provided that if $g$ is an element of $G$ there exists an element of $H$ which contains $g$.

Definition. $A$ point set is said to be peripherally separable provided that its boundary is separable.

Let $S$ denote a locally connected metric space.

1 Presented to the Society, February 22, 1941.

2 See F. B. Jones, Concerning normal and completely normal spaces, this Bulletin, vol. 43 (1937), pp. 671-677.

${ }^{3}$ For the definition of certain terms and phrases, the reader is referred to R. L. Moore's Foundation of Point Set Theory, American Mathematical Society Colloquium Publications, vol. 13, New York, 1932, or to W. Sierpinski's Introduction to General Topology, Toronto, 1934, translated by C. C. Krieger. 